AperTO - Archivio Istituzionale Open Access dell'Università di Torino

An Inventory of Problems-29 Sensitivity Study Investigating Feigning of Four Different Symptom Presentations Via Malingering Experimental Paradigm

This is a pre print version of the following article:

Original Citation:

Availability:

This version is available http://hdl.handle.net/2318/1766445

since 2021-01-12T14:16:26Z

Published version:

DOI:10.1080/00223891.2019.1566914

Terms of use:

Open Access

Anyone can freely access the full text of works made available as "Open Access". Works made available under a Creative Commons license can be used according to the terms and conditions of said license. Use of all other works requires consent of the right holder (author or publisher) if not exempted from copyright protection by the applicable law. 


\section{An Inventory of Problems - 29 (IOP-29) Sensitivity Study Investigating Feigning of Four Different Symptom Presentations via Malingering Experimental Paradigm}

The Inventory of Problems - 29 (IOP-29; Viglione, Giromini \& Landis, 2017) is a new, brief, self-report measure designed to assist practitioners evaluating the credibility of various symptom presentations. It is comprised of 29 items that are administrable via classic, paper-andpencil format, or online, using either a tablet or a PC. Most of the items focus on the subjective experience of the test-taker dealing with his or her problems, some others address specific symptoms or deficits, and a few others present calculation and reasoning problems. By analyzing and combining the responses to each of these 29 items, a logistic regression-derived formula generates the False Disorder Probability Score (FDS), a probability value reflecting the likelihood of drawing that specific IOP-29 from a group of experimental feigners versus a group of bona fide patients, if the a-priori expectations were $50 \%$ to $50 \%$. When the FDS is zero, the presentation is completely credible; when it approaches one, it is not credible at all. More generally, the lower the FDS, the higher the credibility of the presented complaints.

Initial research with the IOP-29 has shown very promising findings. In a series of clinical comparison, simulation studies (experimental malingering paradigm) conducted by Viglione et al. (2017), the classification accuracy of the IOP-29 compared favorably to that of the Test of Memory Malingering (TOMM; Tombaugh, 1996) when testing bona fide versus feigned depression-related presentations $(n=88)$, and resembled to that of the longer and more complex Minnesota Multiphasic Personality Inventory (MMPI-2; Green, 1991) and Personality Assessment Inventory (PAI; Morey, 1991, 2007) when testing bona fide versus feigned schizophrenia- $(n=178)$, depression- $(n=85)$ and mTBI/PTSD-related $(n=128)$ presentations. 
In all these cases, the FDS of the IOP-29 demonstrated sensitivity and specificity of about .80 with the same cut-off score of FDS $\geq .50$, which represents the optimal balance of sensitivity and specificity when there are no a-priori expectations. More recently, Giromini, Viglione, Pignolo and Zennaro (2018) compared the validity of the IOP-29 also against that of the Structured Inventory of Malingered Symptomatology (SIMS; Smith \& Burger, 1997; Widows \& Smith, 2005). They did so by administering both measures to 216 bona fide patients (89 suffering psychotic-related problems, and 127 suffering anxiety, depression, and/or trauma related problems) and to 236 nonclinical participants instructed to feign various psychopathological conditions via malingering experimental paradigm, so to compare classification accuracy statistics. In line with Viglione et al.’s (2017) findings, the IOP-29 performed similarly well with both symptom presentations, with sensitivity and specificity approximating .80 when considering FDS $\geq .50$ as cut-off. Perhaps more importantly, the IOP-29 outperformed the SIMS, with the differences between the two instruments becoming particularly evident especially when considering psychotic-related presentations.

The fact that the IOP-29 was similarly accurate when assessing the credibility of notably different symptom presentations, such as depression, mTBI, PTSD, and psychosis, is in line with the authors' goal of developing a tool that would be applicable to a very wide range of evaluation contexts. In the IOP-29's official website (www.iop-test.com), authors Viglione and Giromini refer to their measure as an "omnibus test," to highlight that the IOP-29 should be suitable and generalizable to many different types of psychiatric and cognitive disorders and their combinations. Moreover, in several passages of their published work, Viglione et al. (2017), Giromini et al. (2018) and Viglione et al. (2018) emphasize that across the multiple research refinements leading up to the development of the IOP-29, they paid particular attention to 
incremental validity, to ensure that each of the items eventually included in the IOP-29 would provide important, non-redundant information. That is, the IOP-29's capability to assess the credibility of different symptom presentations is a key aspect of the IOP-29 project.

Nonetheless, since the IOP-29 is still a relatively new test, additional research on its generalizability from one condition or context to another would certainly be beneficial. In particular, as noted by Giromini et al. (2018), “generalization to cognitive and neuropsychological disorders has limited research support so far, so that both simulation and known-group studies with this population are needed” (p. 9).

Another poorly investigated topic, related to IOP-29 research, is whether the FDS would be sensitive (or not) to uncooperative, resistant or elusive responding. In 1989, Nichols, Greene and Schmolck conceptualized two primary categories of response distortions: content-related and content-unrelated. The former refers to the fact that the test-taker voluntarily endorses response options that are not truthful, because he or she wants to manipulate the impression that the evaluator will make about his or her condition when interpreting the scores of the test. The latter refers to a pattern of endorsement that may result from distraction, random responding, stimulus avoidance, and other similar behaviors that generate various forms of distortion and error variance in the scores. Given the high stakes of forensic contexts, pure random responding is unlikely to happen in forensic evaluations. However, in some cases malingerers may be uncooperative, resistant or elusive, and might therefore opt for a 'random-like’ responding approach. That is, they may choose to respond with poor effort or attention, partially at random, or in a resistant way, not to discover any important information about themselves, i.e., not to truly open themselves to the examiner. To our knowledge, whether or not the IOP-29 FDS 
would detect also this type of random-resistant, uncooperative responding has never been investigated, so far.

\section{The Current Study}

The current study had two major goals. First, we wanted to test the sensitivity of the IOP-29 to feigning of four different, categories of symptom presentations, i.e., depression-, mTBI-, PTSD-, and schizophrenia-related disorders. Second, we wanted to test the sensitivity of the IOP-29 to uncooperative or random-resistant responding. To do so, we recruited a sample of 400 Italian nonclinical volunteers and administered the Italian version of the IOP-29 (Giromini et al., 2018), three times, to each participant, in three different conditions. More in detail, participants were instructed, in one condition, to respond to the IOP-29 items honestly (HON); in one condition, to respond by trying to feign a psychiatric or cognitive disorder (SIM); in one condition to respond in an uncooperative, resistant, random-resistant, or elusive way, as if they did not want to truly answer the questions (RND). Additionally, four different types of instructions were used for conditions SIM and RND, for four subgroups of participants: 100 were instructed to imagine they wanted to convince the examiner they suffered depressionrelated symptoms, 100 that they suffered mTBI-related symptoms, 100 that they suffered PTSDrelated symptoms, and 100 that they suffered schizophrenia-related symptoms. For condition RND, they were instructed to do so by responding randomly, or in an elusive or not organized way.

Our major hypothesis was that there would be small between-group differences in the FDS scores, with participants' FDS scores being notably higher in condition SIM compared to condition HON for all four symptom presentations. Such finding would support the generalizability and applicability of the IOP-29 to different diagnoses and conditions, in line with 
An IOP-29 Sensitivity Study

Viglione et al's (2017) and Giromini et al.’s (2018) indications. We also speculated that because uncooperative, resistant, or random-resistant responding is more unlikely to occur with honest responders than with malingerers, the FDS values in condition RND would locate somehow halfway between those found in conditions HON and SIM, but closer to those in condition SIM than to those in condition HON. Said differently, we anticipated that random-resistant responses would probably resemble more closely those of experimental feigners rather than those of honest responders.

\section{Method}

\section{Participants}

An Italian, community sample comprised of 400 adult volunteers, ranging in age from 18 to $70(M=41.26, \mathrm{SD}=14.31$; age information was missing for two cases $)$ and fairly balanced by gender (56.25\% women) contributed to this study. In terms of education, a little more than half (60.25\%) had a high school degree or less, $15.00 \%$ had a bachelor's degree, and the remaining 25.75\% had a master's degree or more. About half (51.75\%) were currently in a relationship (married and/or cohabiting with a partner), about half (48.25\%) were not in a relationship (single, widowed or divorced). As reported in Table 1, participants instructed to feign depression-, mTBI-, PTSD-, or schizophrenia-related symptoms did not differ from each other in terms of age or gender. Yet, those in the mTBI group tended to have a higher level of education compared to those in the other three groups, and the PTSD group included more individuals who were currently in a relationship, compared to the other three groups. It should be noted, however, that additional analyses conducted after testing the main hypotheses of this study revealed that these dishomogeneities had no impact on the overall findings presented below.

\section{Procedures}


After receiving formal approval by the pertinent institutional review board, data recruitment began by posting advertisements online and via snowball sampling. To maximize generalizability to real-life situations, rather than focusing on college students, we instructed our research assistants to contact any prospective participants outside the academic environment, so to recruit adult individuals of various ages and with various educational levels. Inclusion criteria required to be able to read and sign an informed consent form, not to have any diagnoses of psychiatric or cognitive disorders, and not to be familiar with the IOP-29 items. Participants were always met and administered the IOP-29 individually, one at a time. Each was assigned to one of the four feigning groups randomly, and data collection for each specific group ended when its sample size reached $n=100$.

Once obtained signed informed consent, participants were instructed that they would have to take a brief test of psychological problems three times, assuming a different role at each time. In each condition, prior to administering the IOP-29, participants were asked to carefully read the condition-specific instructions, which consisted of about two A4 pages. The order of administration of the three condition was randomized and counterbalanced across participants. In the "honest" condition (HON), participants were asked to answer the items of the test honestly. In the "simulator" condition (SIM), they were asked to take the test as if they wanted to convince the examiner that they were suffering symptoms from a specific cognitive or psychiatric disorder. To facilitate feigning, they were given a list of symptoms characterizing that specific disorder, along with a description of a real-life scenario - adjusted from Viglione et al. (2017) - in which a person might be motivated to feign that specific disorder (Rogers \& Gillard, 2011). Additionally, they also were warned to 'not over-do it,' or else their performance would not be believable, and they will just look like someone trying to fake a disorder, rather 
like a person genuinely experiencing it (Viglione et al., 2001). Furthermore, they were also informed that if they were able to successfully look like a bona fide patient, and not like a simulator, they could receive a small monetary compensation. Lastly, in the "random-resistant" condition (RND), participants were instructed to take the test as if they were mentally ill and had been asked to take a test to evaluate whether they actually had the right to receive monetary compensation. Importantly, they were asked to pretend they did not want to undergo any psychological evaluations because they were concerned that by revealing themselves they could lose their right to receive compensation, and that therefore they decided to respond the IOP-29 items with an uncooperative, resistant, or random-resistant approach. Also in this case, they were told to not over-do it, or else it would be immediately evident that they simply responded without even reading the questions. And also in this case participants were given a brief description of the target disorder they were imaginarily experiencing when they decided to respond with an evasive, resistant, or random-resistant way.

\section{Data Analysis}

As noted above, the major goals of this study were to test whether the IOP-29 FDS would be similarly sensitive to feigning of four different symptom presentations via malingering experimental paradigm, and whether it would be elevated also by uncooperative or randomresistant responding. Thus, we first tested a mixed, 4 (between-subject factor, symptom presentation: depression, mTBI, PTSD, and schizophrenia) by 3 (within-subjects factor, condition: HON, SIM, and RND) ANOVA, with the IOP-29 FDS as our dependent variable. Next, we focused on diagnostic efficiency statistics and tested the sensitivity and specificity of the IOP-29 by inspecting various cut-off scores. More specifically, since this study did not include any clinical samples, our attention mainly focused on sensitivity, rather than on 
specificity. As such, in addition to the standard threshold of FDS $\geq .50$ (Viglione et al., 2017), we also used FDS $\geq .30$ and FDS $\geq .15$, which in Giromini et al. (2018) yielded sensitivity of .90 and .95 , respectively.

\section{Results}

Table 2 and Figure 1 summarize the distribution of the IOP-29 FDS values across the four symptom presentations and three conditions under investigation. From a technical standpoint, the interaction effect (condition x symptom presentation) was not statistically significant, $p=.054$, though $p$ almost approached the significance threshold of .050. A closer examination Table 2 and Figure 2 indeed reveals that on average, in condition SIM, the FDS was slightly lower for the PTSD-related presentation, compared to the other three symptom presentations, mainly because fewer cases produced extremely high FDS values. The size of the difference between the HON and SIM conditions, however, was very high for all symptom presentations, ranging from $d=2.50$, for the PTSD group, to $d=4.32$, for the depression group. Cohen's $d$ for the entire sample, comparing HON versus SIM, was 3.23 (all these Cohen’s $d$ effect sizes were calculated using standard formula per independent samples, in line with Dunlap et al.’s (1996) recommendations). Along the same lines, receiver operator characteristic curve (AUC) values contrasting conditions HON versus SIM ranged from .94 (SE = .02), for the PTSD group, to .99 ( $S E=.01)$, for the depression group, and was .96 $(S E=.01)$ for the entire sample.

As expected, the average value of the IOP-29 FDS in the RND condition was located halfway between that observed in the HON and SIM conditions. With minimal variations from one symptom presentation to another, uncooperative, resistant, or random-resistant responding produced an average IOP-29 FDS value of .65 $(S D=.27)$, which is about one standard deviation above the standard threshold of FDS $=.50$ for evaluating the credibility of a symptom 
presentation with no a-priori expectations. On average, thus, these records resembled more closely those of experimental simulators rather than those of bona fide patients.

Lastly, we evaluated the classification accuracy of the IOP-29 by inspecting three different cut-off scores that in previous research (Giromini et al., 2018) showed, respectively, sensitivity values of .80 (FDS $\geq .50), .90(\mathrm{FDS} \geq .30)$, and .95 (FDS $\geq .15)$. In the current study, these same threshold scores produced even higher sensitivity values. When considering the entire sample, sensitivity was .91 for FDS $\geq .50$ (i.e., 363 true positives out of 400), .96 for FDS $\geq .30$ (i.e., 385 true positives out of 400 ), and .98 for FDS $\geq .15$ (i.e., 391 true positives out of 400). Importantly, sensitivity estimates did not significantly differ from one symptom presentation to another, ranging from .86 to .95 for FDS $\geq .50$, from .93 to .99 for FDS $\geq .30$, and from .95 to 1.00 for FDS $\geq .15$ (Table 3). In all cases, the PTSD group produced the lowest sensitivity values, the depression group the highest. Also noteworthy, about $70 \%$ of the randomresistant presentations had FDS $\geq .50$, about $85 \%$ had FDS $\geq .30$, and about $90 \%$ had FDS $\geq .15$, with minimal variations from one symptom presentation to another. These data concur to indicate that random-resistant records resembled more closely those of experimental simulators rather than those of bona fide patients, in line with our hypotheses.

Although not the focus of the study, Table 3 also reports on the specificity of the IOP-29 with honest, nonclinical responders. When considering the entire sample, specificity was .93 with FDS $\geq .50, .76$ with FDS $\geq .30$, and .47 with FDS $\geq .15$. Not surprisingly, these values are slightly higher than those reported by Viglione et al. (2017) and Giromini et al. (2018) with data from bona fide patients.

\section{Discussion}


The major goal of this study was to test the sensitivity of the recently developed, Inventory of Problems - 29 (IOP-29; Viglione, Giromini \& Landis, 2017) in detecting feigning of four different symptom presentations via malingering experimental paradigm. By investigating a sample of 1,200 IOP-29 records from 400 nonclinical volunteers, our research provided additional evidence supporting the validity of the IOP-29, and its applicability to multiple contexts and situations. Additionally, it also tested - for the first time, to our knowledge - the extent to which uncooperative or random-resistant responding would elevate the chief feigning scale of the IOP-29, i.e., the False Disorder Probability Score (FDS). Consistent with our expectations, random-resistant responding elevated the FDS more than did honest responding, but less than did experimental feigning of cognitive or psychiatric illness.

The most remarkable finding of this study is that regardless of whether the test-taker feigned, via experimental malingering paradigm, depression-, mTBI-, PTSD-, or schizophreniarelated symptoms, the IOP-29 FDS consistently showed notable elevations, with mean values ranging from $.76(S D=.24)$ to $.86(S D=.16)$, and sensitivity ranging from .86 to .95 when the apriori cut-off of FDS $\geq .50$ was adopted. These data are perfectly in line with previously reported, research findings (Giromini et al., 2018; Viglione et al., 2017), and therefore suggest that the IOP-29 is likely applicable and generalizable to multiple contexts and evaluations, with minimal variations in its validity. Given the marginally significant effect of the interaction between condition and symptom presentation, however, future studies might further test the classification accuracy of the IOP-29 to assess PTSD-related complaints. Perhaps more importantly, future research should attempt to replicate our findings also by including clinical participants. Indeed, our effect size (ranging from $d=2.50$ to $d=4.32$ ) and AUC (ranging from .94 to .99) values concerning the comparison between the honest and feigning conditions were 
likely boosted by the fact that the control IOP-29's did not come from bona fide patients, but from nonclinical participants only (for background and details, see: Rogers et al., 2003; van Impelen et al., 2014).

Interestingly, using the same cut-off scores recommended by Giromini et al. (2018) to obtain sensitivity levels of .80 (FDS $\geq .50$ ), .90 (FDS $\geq .30$ ), and .95 (FDS $\geq .15$ ), yielded in this study average sensitivity values of $.91, .96$, and .98 , respectively. The fact that our participants were given a detailed list of symptoms and a real-life scenario to help them assuming the role of experimental feigners, that they were warned not to over-do it, and that they were motivated by the possibility to receive a small monetary compensation, makes it difficult to understand the reasons for this increased sensitivity. These experimental procedures are indeed virtually identical to those used by Giromini et al. (2018) and Viglione et al. (2017), and are consistent with standard practice for conducting simulation studies (Rogers \& Bender, 2013, 2018; Rogers \& Gillard, 2011; Viglione et al., 2001). The demographic composition of our sample also was not dramatically different from that of simulators included in Giromini et al. (2018), and both researches, i.e., ours and Giromini et al.’s (2018), were conducted using the same, Italian adaptation of the IOP-29. Accordingly, the sensitivity of the IOP-29 FDS might perhaps be slightly higher than hypothesized before, or alternatively this small variation might be simply due to some specific idiosyncrasies of the two samples under investigation.

Another crucial contribution of this article is that we investigated the sensitivity of the FDS to uncooperative or random-resistant responding. Although unlikely to happen with a brief test comprised of 29 items only, random responding is a threat to the validity of self-report measures (Nichols et al., 1988; Osborne \& Blanchard, 2011; Pinsoneault, 2007), and most assessment instruments include specific scales to assess possible random responding (e.g., 
Archer \& Elkins, 1999; Bagby et al., 1991; Charter \& Lopez, 2003; Fronczyk, 2014; Keeley et al., 2016; Kelley et al., 2017). At the moment, a scale to detect random responding with the IOP29 has not yet been developed, so we wanted to test the extent to which the IOP-29 FDS would be elevate if the test-taker responded with a random-resistant style. With this regard, however, it should be noted that our participants were not instructed to respond purely at random: they were asked to respond in an uncooperative, haphazard, elusive, resistant way, as if they did not really want to answer the questions of the test. Methodologically, this approach is very different from most classic studies on random responding, in which data are either generated randomly by a computer (e.g., Archer \& Elkins, 1999; Fronczyk, 2014; Kelley et al., 2017), or collected by providing the participants with the response sheet only, without giving them access to the booklet of the test (e.g., Baer et al., 1999). To some extent, it is also different from other studies in which participants were given the actual test, with the instruction to respond completely at random (e.g., Bagby et al., 1991). In our study, indeed, rather than targeting random responding, we were looking at resistant, reticent, evasive, elusive, haphazard and/or uncooperative responding - and this is the reason why we refer to this style as “random-resistant” responding, rather than simply as "random responding." This approach to attending to the items of a test might perhaps be conceived of as located halfway between a feigning, and a random responding style.

As expected, when participants were asked to respond with this random-resistant approach, their FDS was closer to that of experimental feigners than to that of bona fide patients, reaching an average value of $.65(S D=.25)$. Importantly, when adopting the standard IOP-29 cut-off to investigate the credibility of a symptom presentation with no a-priori expectations, i.e., FDS $\geq .50$ (Giromini et al., 2018; Viglione et al., 2017), sensitivity ranged between .68 and .73. 
This sensitivity is obviously less than optimal, if one aims at detecting, specifically, haphazard, uncooperative, and/or random-resistant responding styles. As such, rather than relying only on the IOP-29 FDS, future refinements of the test should attempt to develop a new IOP-29 scale, possibly embedded in the already available, 29 IOP-29 items, to specifically detect this type of response-style.

In terms of limitations, a few deserve mentioning. First, our using of one vignette only per symptom presentation may have limited our ability to evaluate the impact of minor vignettespecific characteristics on the FDS scores. For example, our PTSD-related presentation used one vignette only, describing a person who lost his house during an earthquake and who is now experiencing PTSD-related symptoms. Future studies might attempt to use other PTSD-related vignettes (e.g., related to tsunami, terrorist attacks, etc.) so to evaluate the extent to which the IOP-29 FDS may vary as a function of these minor, vignette-specific characteristics. Second, like it is true for all simulation studies, the external validity of our study may be questioned, as there may be notable differences between the approaches one decides to use when feigning a disorder in a research context versus in a real-life situation (Rogers \& Benders, 2018). Third, given that we did not use any other tests to evaluate the validity of a symptom presentation, aside from the IOP-29, we could not evaluate the extent to which our experimental feigners may or may not be representative of 'prototypical' experimental feigners. Fourth, although the administration order was randomized and counterbalanced across conditions, the fact that the same person completed the same test three times, albeit with different instructions, may have added some uncontrolled, error variance to the experiment. It should be noted, however, that this hypothetical 'noise' was equally distributed across all four subsamples, so that it should not affect our main finding that the IOP-29 FDS was remarkably sensitive to all four different 
An IOP-29 Sensitivity Study

symptom presentations. Finally, the four subgroups assigned to the four different symptom presentations were not perfectly balanced in terms of educational level and relationship status. Albeit our post-hoc analyses controlling for these variables yielded basically the same results as those presented in this paper, future studies should attempt to replicate our findings by using more balanced samples with regards to these two variables. Despite all these limitations, the current study still has the merit to have provided some additional data supporting the applicability of the IOP-29 to various symptom presentations, and to have presented some data concerning the sensitivity of the IOP-29 FDS to uncooperative or random-resistant responding. 


\section{References}

Archer, R. P., \& Elkins, D. E. (1999). Identification of random responding on the MMPI-A. Journal of Personality Assessment, 73, 407 -421

Baer, R. A., Kroll, L. S., Rinaldo, J., \& Ballenger, J. (1999). Detecting and discriminating between random responding and overreporting on the MMPI-A. Journal of Personality Assessment, 72(2), 308-320.

Bagby, R. M., Gillis, J. R., Toner, B. B., \& Goldberg, J. (1991). Detecting fake-good and fakebad responding on the Millon Clinical Multiaxial Inventory-II. Psychological Assessment, 3, 496-498.

Charter, R. A., \& Lopez, M. N. (2003). MMPI-2: Confidence intervals for random responding to the F., F Back, and VRIN scales. Journal of Clinical Psychology, 59(9), 985-990.

Fronczyk, K. (2014). The identification of random or careless responding in questionnaires: The example of the NEO-FFI. Roczniki Psychologiczne, 17(2), 459-473.

Giromini, L., Viglione, D.J., Pignolo, C. \& Zennaro, A. (2018). A clinical comparison, simulation study testing the validity of SIMS and IOP-29 with an Italian sample. Psychological Injury and Law, advance online publication, doi:10.1007/s12207-0189314-1

Green, R. L. (1991). MMPI-2/MMPI: An interpretative manual. Boston, MA: Allyn \& Bacon.

Keeley, J. W., Webb, C., Peterson, D., Roussin, L., \& Flanagan, E. H. (2016). Development of a response inconsistency scale for the Personality Inventory for DSM-5. Journal of Personality Assessment, 98, 351-359. doi:10.1080/00223891.2016.1158719 
Kelley, S. E., Edens, J. F., Donnellan, M. B., Somma, A., Fossati, A. , de Ruiter, C., Eisenbarth, H., \& Vaughn, M. G. (2017). Detecting inconsistent responding on the Youth Psychopathic Traits Inventory-Short Form. Assessment, Advance Online Publication.

Morey, L. C. (1991). Personality Assessment Inventory. Professional manual. Odessa, FL: Psychological Assessment Resources.

Morey, L. C. (2007). Personality Assessment Inventory (PAI). Professional manual (2nd ed.). Odessa, FL: Psychological Assessment Resources.

Nichols, D. S., Greene, R. L., Schmolck, P. (1989). Criteria for assessing inconsistent patterns of item endorsement on the MMPI: Rationale, development, and empirical trials. Journal of Clinical Psychology, 45(2), 239-250.

Osborne, J. W., \& Blanchard, M. R. (2011). Random responding from participants is a threat to the validity of social science research results. Frontiers in Psychology, 1, 220.

Pinsoneault, T. B. (2007). Detecting random, partially random, and nonrandom Minnesota Multiphasic Personality Inventory-2 protocols. Psychological Assessment, 19(1), 159-164

Rogers, R. \& Bender, D. (2018). Clinical assessment of malingering and deception. New York, NY: Guilford Press.

Rogers, R., \& Bender, S. D. (2013). Evaluation of malingering and related response styles. In I. B. Weiner (Ed.-in-Chief), J. R. Graham, \& J. A. Naglieri (Vol. Eds.), Comprehensive handbook of psychology: Assessment psychology (2nd ed., Vol. 10, pp. 517-540). Hoboken, NJ: Wiley.

Rogers, R., \& Gillard, N. D. (2011). Research methods for the assessment of malingering. In B. Rosenfeld \& S. Penrod (Eds.), Research methods in forensic psychology (pp. 174-188). Hoboken, NJ: Wiley. 
Rogers, R., Sewell, K. W., Martin, M. A., \& Vitacco, M. J. (2003). Detection of feigned mental disorders: A meta-analysis of the MMPI-2 and malingering. Assessment, 10, 160-177.

Smith, G. P., \& Burger, G. K. (1997). Detection of malingering: Validation of the Structured Inventory of Malingered Symptomatology (SIMS). Journal of the American Academy on Psychiatry and Law, 25, 180-183.

Tombaugh, T. N. (1996). Test of Memory Malingering (TOMM). North Tonawanda, NY: MultiHealth Systems.

van Impelen, A., Merckelbach, H., Jelicic, M., \& Merten, T. (2014). The Structured Inventory of Malingered Symptomatology (SIMS): A systematic review and meta-analysis. The Clinical Neuropsychologist, 28, 1336-1365.

Viglione, D. J., Giromini, L. \& Landis, P. (2017). The development of the Inventory of Problems-29: A brief self-administered measure for discriminating bona fide from feigned psychiatric and cognitive complaints. Journal of Personality Assessment, 99, 534-544.

Viglione, D. J., Giromini, L., Landis, P., McCullaugh, J.M., Pizitz, T.D., O'Brien, S., Wood, S., Connell, K., \& Abramsky, A. (2018). Development and Validation of the False Disorder Score: The Focal Scale of the Inventory of Problems. Journal of Personality Assessment, Advance Online Publication

Viglione, D. J., Wright, D., Dizon, N. T., Moynihan, J. E., DuPuis, S., \& Pizitz, T. D. (2001). Evading detection on the MMPI-2: Does caution produce more realistic patterns of responding? Assessment, 8, 237-250.

Widows, M. R., \& Smith, G. P. (2005). SIMS-Structured Inventory of Malingered Symptomatology. Professional manual. Lutz, FL: Psychological Assessment Resources. 
An IOP-29 Sensitivity Study

Table 1. Composition of the Sample.

\begin{tabular}{|c|c|c|c|c|c|}
\hline & \multicolumn{4}{|c|}{ Feigned Symptom Presentation } & \multirow{2}{*}{$\begin{array}{l}\text { Entire Sample } \\
\qquad(n=400)\end{array}$} \\
\hline & $\begin{array}{c}\text { Depression } \\
(n=100)\end{array}$ & $\begin{array}{c}\text { mTBI } \\
(n=100)\end{array}$ & $\begin{array}{c}\text { PTSD } \\
(n=100)\end{array}$ & $\begin{array}{l}\text { Schizophrenia } \\
\quad(n=100)\end{array}$ & \\
\hline \multicolumn{6}{|l|}{ Age $[F(3,394)=326.10, p=.19]$} \\
\hline$M$ & 41.55 & 40.51 & 43.64 & 39.37 & 41.26 \\
\hline$S D$ & 15.18 & 14.66 & 10.92 & 15.79 & 14.31 \\
\hline \multicolumn{6}{|l|}{ Gender $\left[\operatorname{Chi}^{2}(3)=4.09, p=.25\right]$} \\
\hline$M$ & 36 & 45 & 44 & 50 & 175 \\
\hline$F$ & 64 & 55 & 56 & 50 & 225 \\
\hline \multicolumn{6}{|l|}{ Education $\left[\operatorname{Chi}^{2}(6)=43.25, p<.01\right]$} \\
\hline High school or less & 59 & 35 & 76 & 71 & 241 \\
\hline Bachelor's degree & 12 & 23 & 11 & 10 & 56 \\
\hline Master's degree or more & 29 & 42 & 13 & 19 & 103 \\
\hline \multicolumn{6}{|l|}{ Relationship status [Chi $\left.{ }^{2}(3)=15.49, p<.01\right]$} \\
\hline Not in a relationship (single, divorced, ...) & 49 & 55 & 32 & 57 & 193 \\
\hline In a relationship (married, cohabiting) & 51 & 45 & 68 & 43 & 207 \\
\hline
\end{tabular}

Note. Age information was missing for two cases. 
Table 2. IOP-29 FDS Values across the Four Symptom Presentations and Three Conditions: Descriptive Statistics

\begin{tabular}{|c|c|c|c|c|c|}
\hline & \multicolumn{4}{|c|}{ Feigned Symptom Presentation } & \multirow{2}{*}{$\begin{array}{l}\text { Entire Sample } \\
\qquad(n=400)\end{array}$} \\
\hline & $\begin{array}{l}\text { Depression } \\
(n=100)\end{array}$ & $\begin{array}{c}\text { mTBI } \\
(n=100)\end{array}$ & $\begin{array}{c}\text { PTSD } \\
(n=100)\end{array}$ & $\begin{array}{l}\text { Schizophrenia } \\
\qquad(n=100)\end{array}$ & \\
\hline \multicolumn{6}{|c|}{ Condition: Honest (HON) } \\
\hline$M$ & .19 & .20 & .23 & .25 & .22 \\
\hline$S D$ & .15 & .16 & .18 & .19 & .17 \\
\hline \multicolumn{6}{|c|}{ Condition: Simulation (SIM) } \\
\hline$M$ & .86 & .81 & .76 & .85 & .82 \\
\hline$S D$ & .16 & .21 & .24 & .19 & .20 \\
\hline \multicolumn{6}{|c|}{ Condition: Random-resistant (RND) } \\
\hline$M$ & .65 & .64 & .64 & .66 & .65 \\
\hline$S D$ & .27 & .28 & .27 & .26 & .27 \\
\hline
\end{tabular}

Note. The interaction effect was marginally significant, $F(6,792)=2.074, p=.054$; the main effect of symptom presentation was nonsignificant $F(3,396)=1.624, p=.183$; the main effect of condition was significant, $F(2,792)=932.210$, $p<.001$, with all pairwise comparisons being significant at $p<.001$ also after Bonferroni correction. 
Table 3. Classification Accuracy of the IOP-29 FDS across the Four Symptom Presentations and Three Conditions

\begin{tabular}{|c|c|c|c|c|c|c|}
\hline & \multicolumn{4}{|c|}{ Feigned Symptom Presentation } & \multirow[b]{2}{*}{$\operatorname{Chi}^{2}(3)$} & \multirow[b]{2}{*}{$p$} \\
\hline & $\begin{array}{l}\text { Depression } \\
(n=100)\end{array}$ & $\begin{array}{c}\text { mTBI } \\
(n=100)\end{array}$ & $\begin{array}{c}\text { PTSD } \\
(n=100)\end{array}$ & $\begin{array}{l}\text { Schizophrenia } \\
\qquad(n=100)\end{array}$ & & \\
\hline \multicolumn{7}{|c|}{ Condition: Honest (HON) } \\
\hline$F D S \geq .50$ & $96 / 4$ & $94 / 6$ & $91 / 9$ & 89 / 11 & 4.18 & .24 \\
\hline$F D S \geq .30$ & 82 / 18 & $80 / 20$ & 72 / 28 & $70 / 30$ & 5.70 & .13 \\
\hline$F D S \geq .15$ & $51 / 49$ & $51 / 49$ & $46 / 54$ & $41 / 59$ & 2.76 & .43 \\
\hline \multicolumn{7}{|c|}{ Condition: Simulation (SIM) } \\
\hline$F D S \geq .50$ & $5 / 95$ & $12 / 88$ & 14 / 86 & $6 / 94$ & 7.00 & .07 \\
\hline$F D S \geq .30$ & $1 / 99$ & 4 / 96 & $7 / 93$ & $3 / 97$ & 5.20 & .16 \\
\hline$F D S \geq .15$ & $0 / 100$ & 3 / 97 & $5 / 95$ & $1 / 99$ & 6.70 & .08 \\
\hline \multicolumn{7}{|c|}{ Condition: Random-resistant (RND) } \\
\hline$F D S \geq .50$ & $27 / 73$ & 32 / 68 & 31 / 69 & $28 / 72$ & .82 & .85 \\
\hline$F D S \geq .30$ & 15 / 85 & 18 / 82 & 13 / 87 & 13 / 87 & 1.33 & .72 \\
\hline$F D S \geq .15$ & 10 / 90 & 7 / 93 & 8 / 92 & 7 / 93 & .82 & .85 \\
\hline
\end{tabular}

Note. Values on the left of the slash indicate negative classifications; values on the right of the slash indicate positive classifications. 
An IOP-29 Sensitivity Study

Figure 1. IOP-29 FDS Values across the Four Symptom Presentations and Three Conditions: Graphical Representation

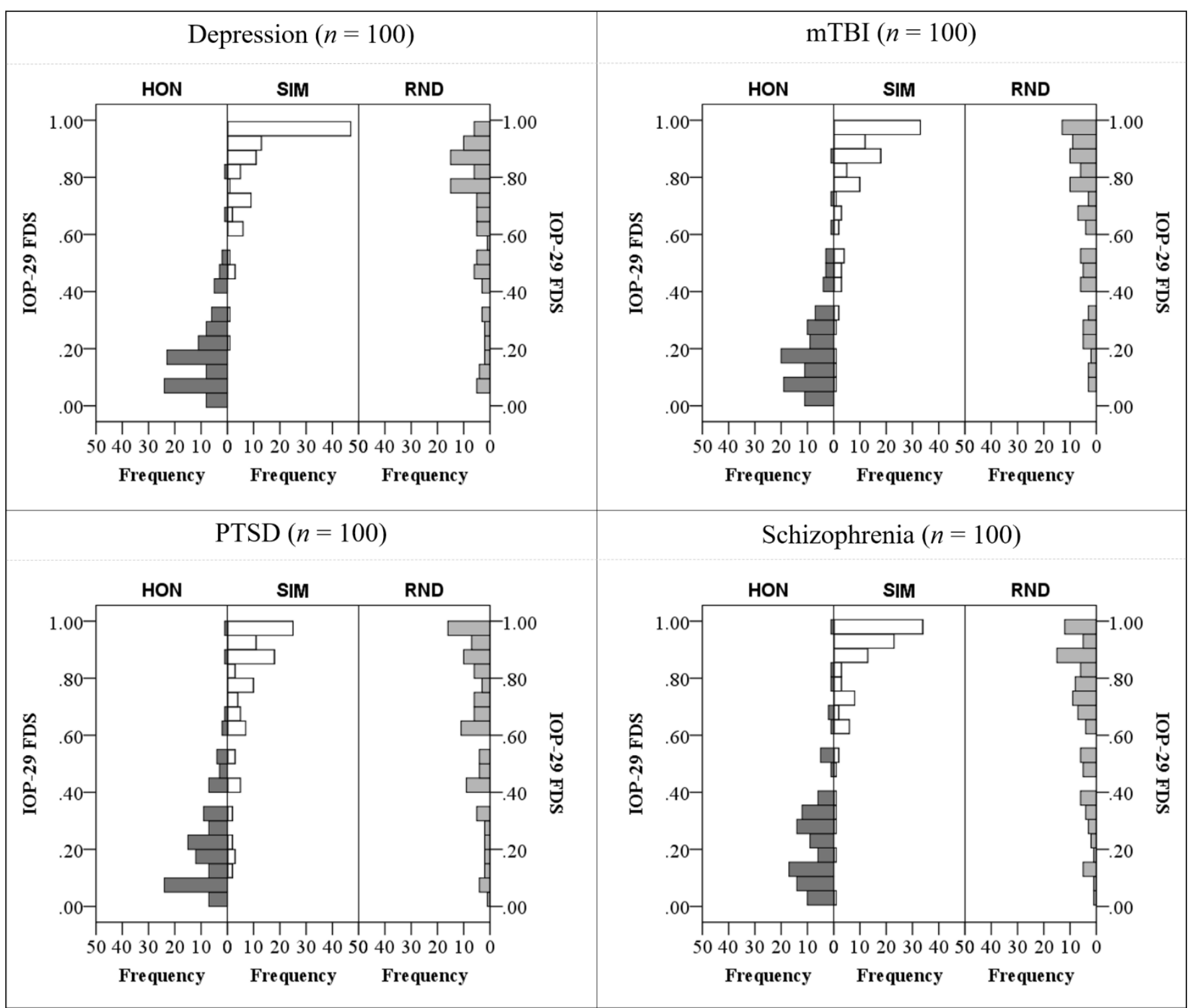

